\title{
ФОРМАЛЬНЫЙ ПОДХОД К АНАЛИЗУ ЛОГИЧЕСКОГО УРОВНЯ ИНТЕРФЕЙСА
}

\author{
(Представил Э. Тьугу)
}

В процессе проектирования цифровых систем на основе стандартных интерфейсов наиболее ответственным и трудоемким этапом является обеспечение логической совместимости сопрягаемых устройств в строгом соответствии с общим протоколом обмена. Недостаточная формализация описания логического уровня интерфейсов приводит к неоднозначной и излишне усложненной трактовке правил обмена. В данной статье делается попытка создания единого подхода к анализу логического уровня существуюших интерфейсов.

Ввиду того, что терминология, используемая при описании логических структур интерфейсов, еще не установилась, раскроем содержание основных терминов и понятий, необходимых для дальнейшего изложения.

Провода, соединяющие устройства, будем называть линиями, а линин, сгруппированные по некоторому функциональному признаку или назначению, - шинами. Совокупность всех линий образует мапистраль интерфейса. Существуют, например, шины данных (состоят, как правило, не менее чем из восьми линий) для передачи информации; шины адресов для осуществления адресации определенного количества устройств заданным способом кодирования; шины управления захватом магистрали для передачи запросов от устройств K арбитру на захват магистрали, подтверждающие и разрешающие сигналы захвата магистрали и т. д. В ряде случаев по одной и той же шине может передаваться разнородная информация, например, данные и адреса, при этом одна или несколько линий шины используются для идентификации признаков передаваемой информации.

Взаимодействие между блоками, подключенными к магистрали, осуществляется путем изменения уровней напряжения на линиях. При описании интерфейса удобно иметь дело не с уровнями напряжений, a c сообщениями. Под сообщением будем понимать наличие на одной или нескольких линиях определенных уровней напряжения. Сообщения могут быть многопроводными и однопроводными. Если некоторый блок А устанавливает на магиетрали уровни напряжения, соответствующие какому-либо сообщению, а блок Б его распознает, то будем говорить, что блок А передал сообщение блоку Б, а блок Б его принял.

Дистанционными будем называть сообщения, пересылаемые по магистрали интерфейса, а местными - сообщения, пересылаемые между интерфейсным блоком (ИБ) и подключенным к нему прибором - устройством ввода-вывода ЭВМ (УВВ). На рис. 1 показан обмен между двумя устройствами, объединенными в систему с помощью интерфейса. Устройства, подключенные к магистрали, обмени- 


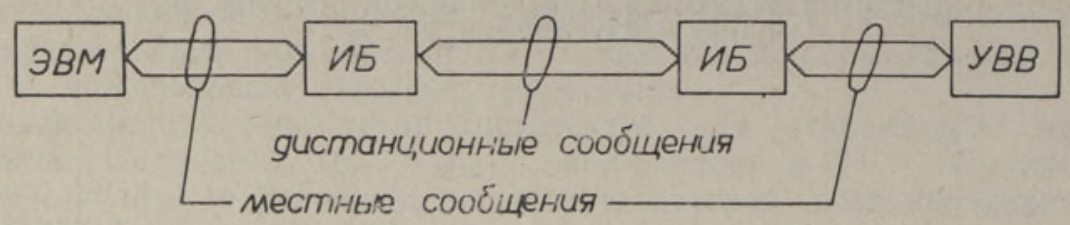

Рис. 1.

ваются дистанционными сообщениями в соответствии с определенным набором правил, называемым протоколом обмена. Обычно принято протокол обмена описывать неформально, например, с помощью разговорного языка или эпюр напряжений на линиях магистрали. Неформальный подход создает иллюзию понятности описания, однако те, кому приходится сталкиваться с конструированием ИБ, знают, сколько вопросов возникает из-за неоднозначного толкования или неполного описания логического уровня интерфейса. Имеется ряд формальных методов описания протоколов, применяемых в сетях ЭВМ, - конечные автоматы $\left[{ }^{1}\right]$, формальные грамматики $\left[{ }^{2}\right]$, сети Петри $\left[{ }^{3}\right]$, логические матрицы $\left[{ }^{4}\right]$ и графы $\left[{ }^{5}\right]$. Эти методы можно использовать и для описания протоколов обмена в интерфейсе.

Формальные методы описания можно разделить на два класса. Описания первого класса представляют каждый из ИБ в виде функции входных сообщений и внутренних состояний, т. е.

$$
\begin{gathered}
M_{\mathrm{Bbx}_{t}}=F_{1}\left(M_{\mathrm{Bx}_{i}} ; m_{\mathrm{Bx}_{i}} ; S_{i}\right), \\
m_{\mathrm{Bbx}_{t}}=F_{2}\left(M_{\mathrm{Bx}_{i}} ; m_{\mathrm{Bx}_{i}} ; S_{i}\right), \\
S_{i+1}=F_{3}\left(M_{\mathrm{Bx}_{i}} ; m_{\mathrm{Bx}_{i}} ; S_{i}\right),
\end{gathered}
$$

где $M$ - множество дистанционных сообщений, $M_{\mathrm{Bx}_{t}}, M_{\mathrm{Bыx}_{t}}-$ входное и выходное дистанционные сообщения $\left(M_{\mathrm{Bx} /}, M_{\mathrm{Bыx}} \in M\right) ; m-$ мно-

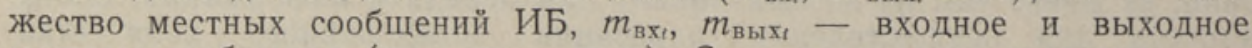
местные сообщения $\left(m_{\mathrm{Bx} t}, m_{\mathrm{выx}} \in m\right) ; S_{i}-$ внутреннее состояние.

В описаниях второго класса магистраль, соединяющая два ИБ, мысленно рассекается и проводится наблюдение за появлением дистанционных сообщений. Их появление в сечении описывается с помощью некоторого автономного автомата

$$
A=\left(M, S, Z, S_{0}, Y\right),
$$

где $M$ - входной алфавит (множество дистанционных сообщений, которыми обмениваются блоки через магистраль), $S$ - множество внутренних состояний автомата, $Z-$ множество конечных состояний автомата, $S_{0}$ - начальное состояние, $Y$ - функция переходов автомата. Описания второго класса не учитывают влияния местных сообщений на появление дистанционных, однако наглядно показывают взаимодействие нескольких блоков. Они могут применяться для описания интерфейсов, в которых не определен набор местных сообщений, или для проверки на соответствие стандарту реализаций интерфейса. Описания первого класса предпочтительны при конструировании специализированных узлов, применяемых в ИБ. В настоящее время только один стандарт на приборный интерфейс МЭК $\left[{ }^{6}\right]$ имеет достаточно полное формальное описание.

Анализ ряда интерфейсных протоколов показывает, что различным интерфейсам присущи наборы общих функций. Как правило, величина 
набора определяется количеством блоков в системе и способом их соединения. Так, в простейших системах, состоящих всего из двух устройств, реализуется функция обмена информацией, иногда дополняемая функцией обмена командами. В более сложных системах набор функций расширяется. Появляются функция захвата магистрали, функция арбнтража (управления захватом магистрали), функция адресации ИБ в системе, функция прерывания. Этот набор зависит также и от прибора, подключенного к интерфейсному блоку. Представим простейшую систему, состоящую из двух устройств: цифрового вольтметра, измеряющего величину напряжения через определенные фиксированные промежутки времени, и перфоратора, регистрирующего результаты измерений на перфоленте. Для построения такой системы требуется только функция обмена информацией. ИБ вольтметра должен обеспечивать выдачу, а ИБ перфоратора - прием информации. Построение системы из модуля отображения информации и ЭВМ потребует добавления функции обмена командами и, возможно, функции прерывания. Увеличение числа цифровых вольтметров потребует введения некоторого блока управления, обеспечивающего адресацию перфоратора и одного из вольтметров, а также выдачу команд на проведение измерения и пробивку результата.

В целях упрощения анализа интерфейса удобно ввести в рассмотрение некоторые простые блоки, в дальнейшем называемые логическими участниками обмена (ЛУО):

приемник - блок, обеспечивающий только прием информации в процессе обмена;

источник - блок, обеспечиваюший только выдачу информации;

контроллер - блок, участвующий в захвате магистрали и управлении адресацией других блоков;

арбитр - блок, управляюший захватом магистрали.

Функция адресации может выполняться любым из ЛУО.

Описания интерфейсных протоколов далее будут выполнены с помощью набора диаграмм состояний функций интерфейса, представляющих собой орграф, вершинам которого соответствуют состояния функции, а дугам - условия переходов из одного состояния в другое. Этимк условиями являются дистанционные сообщения. Рассмотрим способы реализации интерфейсных функций, встречающихся в стандартных интерфейсах, на примере приемника и источника.

\section{Адресация ЛУо в системе}

Взаимная адресация применяется в системах, состоящих из двух ЛУО: приемника и источника. Каждый из них выдает сообщение о своей работоспособности - РП и РИ. При одновременном наличии этих сообщений оба участника переходят в адресованные состояния и могут начинать обмен информацией или командами (рис. 2). Взаимная адресация блоков реализована в интерфейсах ИРПР, BS-4421 [ $\left.{ }^{6}\right]$.

Рис. 2 .

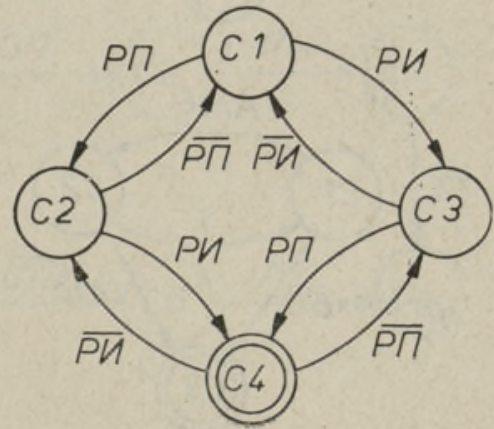




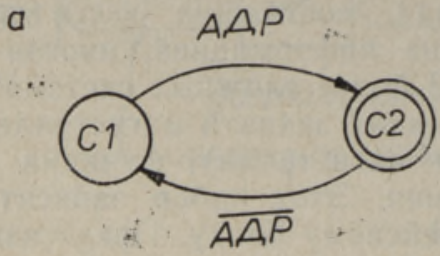

$\delta$

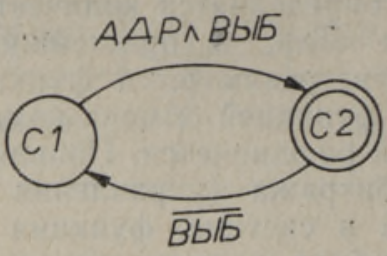

Рис. 3.

Диаграммы состояний для случая прямой адресации показаны на рис. 3. Если каждая линия шины адреса жестко закреплена за какимнибудь блоком, то для его адресации достаточно выдать адресное сообщение без подтверждения. По окончании передачи адресного сообщения блок возвращается в исходное состояние (рис. 3, a). Если адресное сообщение кодируется двоичным набором, то через небольшой промежуток времени после выдачи адреса посылается сообщение, подтверждающее наличие адреса на шине (рис. 3, б). Такое однопроводное сообщение, как правило, называется выборкой или стробом. Необходимость стробирования многопроводных сообщений вызвана тем, что переходные процессы при посылке адресного сообщения могут привести к искажению адреса («перекосу» информации на линиях шины), а следовательно, и к неверной адресации блока. Адресация такого типа применяется в интерфейсах «Общая шина», Multibus и др. $[6,7]$.

В системном интерфейсе ИУС применяется адресация с выдачей блоком квитанции о приеме адреса - адресация с подтверждением (рис. 4, a). При получении своего адреса (сообщение АДР) блок выдает сообщение ППА (подтверждение приема адреса) и переходит в адресованное состояние, в котором продолжает оставаться до тех пор, пока не начнется адресация какого-либо другого устройства (т. е. пока блок не примет чужой адрес - ЧАДР). Если ПППА не появится
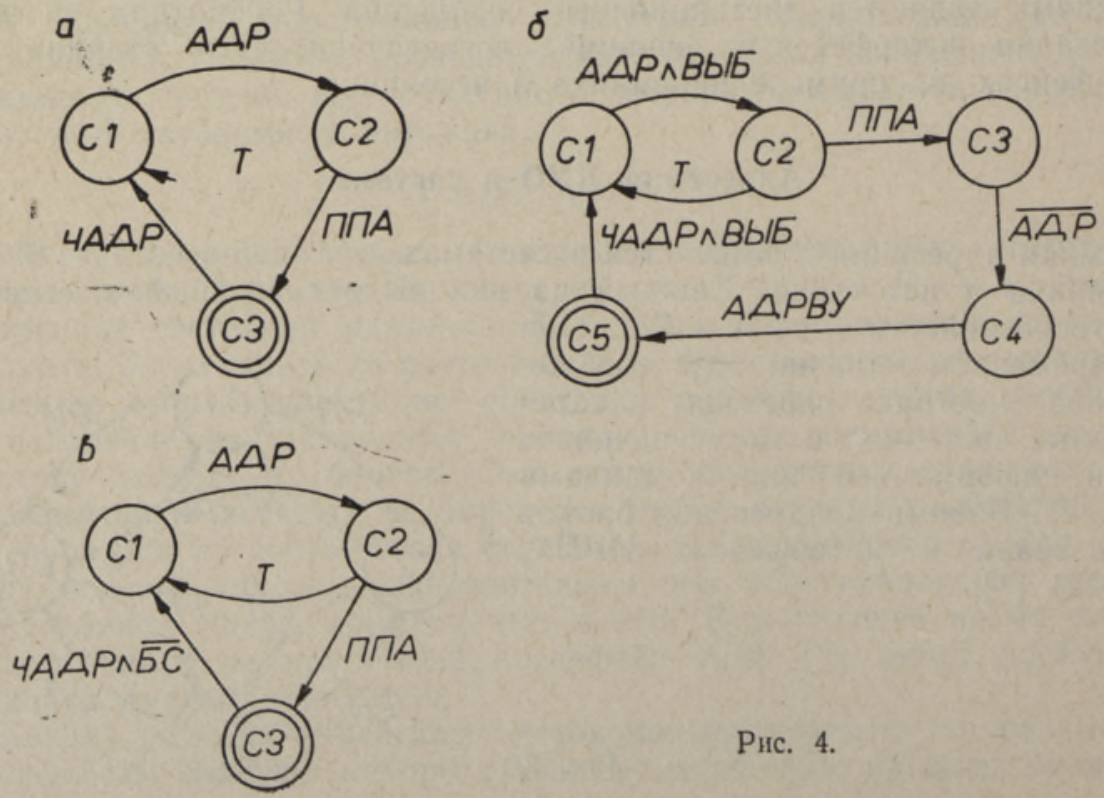

Рис. 4. 
в течение времени $T$, тогда фиксируется тайм-аут и функция адресации возвращается в исходное состояние.

В ЕС ЭВМ применяется иной тип адресации с подтверждением (рис. 4, б). После получения ППА шина адреса освобождается и адресуемое устройство выдает каналу свой адрес (АДРВУ). Таким образом проверяется правильность установления связи с нужным устройством.

В ряде интерфейсов, где требуется одновременная адресация нескольких блоков, для организации обмена между источником и несколькими приемниками применяется блокировка сброса (БС) адресованного состояния. Для передачи этого признака используется либо специальная линия (напр., в интерфейсе ИУС), либо одна из линий адресной шины (приборный интерфейс МЭК). При получении признаков ЧАДР и БС блок продолжает оставаться в адресованном состоянии (рис. 4,8 ).

\section{Обмен данными}

Обмен данными может происходить только между адресованными источниками и приемниками, т. е. функция адресации всегда предшествует функции обмена данными. На практике используются три способа обмена данными, различающихся количеством однопроводных сообщений для организации обмена.

Прямой обмен со стробированием (СТP) - простейший вариант обмена, в котором для передачи данных используется только одно сообщение для подтверждения наличия данных на шине. Источник устанавливает данные на линиях, а через небольшой промежуток вре-
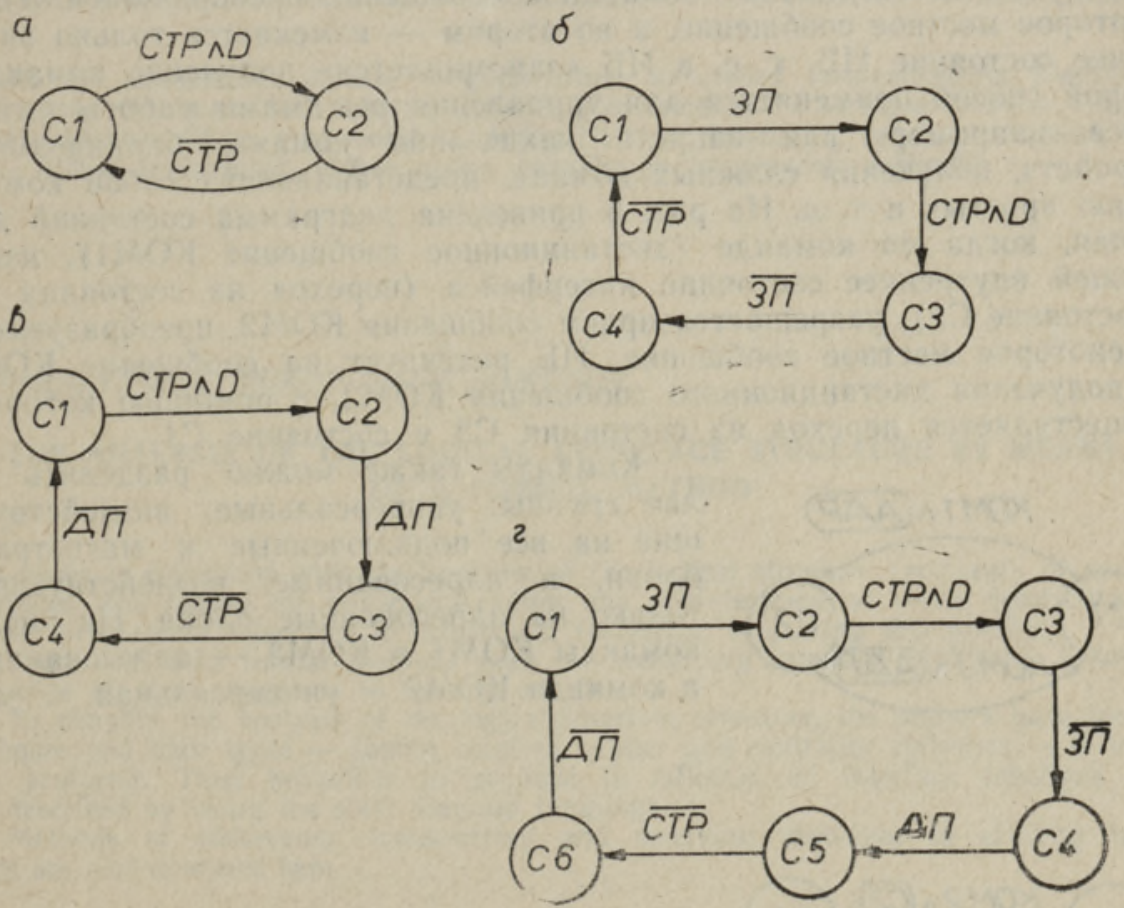

Рис. 5. 
мени, необходимый для ликвидации «перекоса» информации, выдает сообщение СТP в течение фиксированното интервала времени, заведомо большего времени, необходимого для приема данных. Приемник, обнаружив сообщение СТР, считывает информацию (рис. 5, a). Такой способ обмена используется, например, в системе САМАС $\left[{ }^{6}\right]$.

Вариант обмена, называемый двухпроводным «рукопожатием», наиболее распространен в асинхронных интерфейсах. Имеются две разновидности организации двухпроводного «рукопожатия» - по инициативе приемника и по инициативе источника. В первом случае (рис. 5,6$)$ приемник выдает запрос (3П), приняв который, источник устанавливает на шине данные и сообщение СТР, подтверждающее их наличие. Приемник, получив данные, прекращает выдачу сообщения 3П, после чего источник очищает шину данных (прекращается выдача сообщения СТР). Во втором случае (рис. 5, в) адресованный источник выдает данные и сообщение СТР, а приемник, закончив прием, выдает сообщение, что данные приняты (ДП). После этого источник прекращает выдачу информации.

В приборном интерфейсе МЭК реализован способ обмена, называемый трехпроводным «рукопожатием». Этот способ представляет собой сочетание двух описанных выше. В нем используются три сообщения 3П, СТР, ДП (рис. 5,2$)$. Данная схема позволяет организовать асинхронный обмен между одним источником и группой приемников.

\section{Обмен командами}

Имеется два вида реакции ИБ на прием командного сообщения. В первом случае дистанционное командное сообщение преобразуется ИБ в некоторое местное сообщение, а во втором - изменяется только внутреннее состояние ИБ, т. е. в ИБ «запоминается» получение команды. Второй способ применяется для управления режимами работы интерфейса, например, для запрета каких-либо команд, блокирования устройств, получения сложных команд, представляющих собой комбинацию простых и т. д. На рис. 6 приведена диаграмма состояний для случая, когда по команде (дистанционное сообщение KOM1), изменяющей внутреннее состояние интерфейса (переход из состояния С1 в состояние С2), разрешается прием сообщения КОМ2, преобразуемого в некоторое местное сообщение. ИБ реагирует на сообщение КОМ2 до получения дистанционного сообщения KOM3, с помощью которого осушествляется переход из состояния С2 в состояние С1.

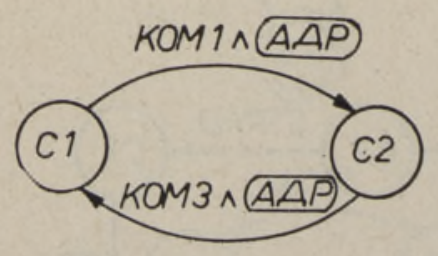

Команды также можно разделить на две группы: универсальные, воздействующие на все подключенные к магистрали блоки, и адресованные, воздействующие только на адресованные блоки. На рис. 6 команды KOM1 и KOM3 - адресованные, а команда КОМ2 - универсальная.

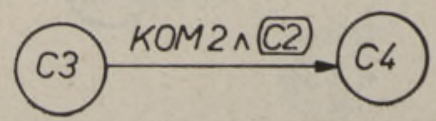

Рис. 6. 


\section{Выводы}

1. Выделены способы адресации устройств, обмена информацией и командами в современных интерфейсах.

2. Предложен формальный подход, пригодный для описания логического уровня любого интерфейса при решении задач анализа и синтеза систем сопряжений.

\section{Л И Т Е Р А Т У Р А}

1. B jorner, D., In: Fall Joint Comput. Conf., AFIPS Conf. Proc., 37, New York City, 1970 , p. $477-491$.

2. Harangozo, J., In: Proc. 5th Data Commun. Symp. Snowbird-Utah, New York, 1977 , p. $4-37-4-49$.

3. Mer 1 i n, P. M., IEEE Trans. Commun., COM-27, № 11, 1671-1680 (1979).

4. Le Moli, G., Alta freq., 42, № 10, 493-500 (1973).

5. D a n thine, A., IEEE Trans. Commun., COM-28, № 4, 632-643 (1980).

6. Х а з а н о Б. И., Интерфейсы измерительных систем, М., «Энергия», 1979.

7. B a rthmaler, J., INTEL MULTIBUS Interfacing, Application Note AP-28A, INTEL Corporation, 1978.

Институт кибернетики Академии наук Эстонской ССР
Поступила в редакцию 13/IV 1981

\section{VARSAVSKI, E. VOROBJOV, V. LOMUNOV}

\section{FORMAALNE LÄHENEMINE LIIDESTE LOOGILISE TASEME ANALOUSILE}

Artiklis on analüüsitud liideste loogilist taset, kasutades formaalkeelena üleminekudiagramme. On vaadeldud vahetusprotokolli pōhielemente - adresseerimisviise, andmeja käsuvaheluse tüüpe ning eeskirju, defineeritud ja kasutatud vahetuse loogilise osavõtja, liidese funktsiooni, liidese oleku, kohalike ja kaugteadete mõisteid.

\section{THE ANALYSIS OF THE LOGICAL INTERFACE STRUCTURE BY MEANS OF A FORMAL METHOD}

This paper is concerned with the analysis of the logical structure (protocol) of present interfaces. The reason for misunderstanding and misinterpretation while describing the logical structure of interfaces is insufficient formalism in solving this problem.

There are some formal methods used for describing interface protocols. A formal language of state diagrams is worked out here.

To simplify the analysis of the logical interface structure, the authors have viewed colloques and their types - source, receiver, arbiter and controller colloques - in interface protocols. Their behaviour in protocol is reflected by interface furctions that are described by using the state diagram language.

Methods of addressing, transmitting and receiving messages in existing interfaces are also analysed here, 